\title{
Comparison of Quality of Life among Patients with Oro-Hypopharyngeal Cancer after Tonsillectomy and Panscopy Using Transoral Robotic Surgery: A Pilot Study
}

\author{
Amir M. Salehi ${ }^{a} \quad$ Lena Norberg-Spaak $^{a}$ Torben Wilms ${ }^{a}$ Simon Vallin ${ }^{b}$ \\ Linda Boldrup $^{c}$ Nicola Sgaramellac ${ }^{c}$ Morad Majlesi ${ }^{d}$ Shahram Nezafat ${ }^{e}$ \\ Karin Nylander ${ }^{\mathrm{c}}$ \\ aDepartment of Otorhinolaryngology and Head and Neck Surgery, Umeå University, Umeå, \\ Sweden; bepartment of Statistics, Registercentrum Norr, Umeå University, Umeå, Sweden; \\ 'Department of Medical Biosciences, Umeå University, Umeå, Sweden; dintuitive Surgical \\ Sàrl, Aubonne, Switzerland; ePC-Allservice, Stockholm, Sweden
}

\section{Keywords}

Quality of life · Transoral robotic surgery · Oro-hypopharyngeal cancer · QLQ-C30 · H\&N35

\begin{abstract}
Studies have shown lower treatment-related morbidity when using transoral robotic surgery (TORS) compared to conventional surgery. Patients investigated for oro- and hypopharyngeal cancer $(T 1, T 2)$ were compared concerning quality of life (QoL) after tonsillectomy and TORS using validated QoL questionnaires: QLQ-C30 and QLQ-H\&N35. The patients treated with TORS showed a higher pain score and thus also a higher need for painkillers, whereas they had lower values on self-assessment of anxiety/depression using the Hospital Anxiety and Depression Scale score. The pre- and postoperative information given did not meet the expectations of the patients treated with conventional surgery. The present data show advantages of the TORS technique from the patients' perspective. Even if patients treated with TORS are in need of more painkilling treatment, they cope better with the long-term effects of treatment, as judged by self-assessment of anxiety and depression.

\section{Introduction}

Treatment of patients with head and neck cancer has changed over the years from predominantly surgical to a combination of therapies such as surgery and pre- or postoperative chemoradiotherapy. Induction chemotherapy or neoadjuvant therapy is sometimes 
Table 1. Patient characteristics and treatment performed

\begin{tabular}{lllc}
\hline Patient characteristics & Total & Male & Female \\
\hline All - mean age (range), years & $64(35-83)$ & $65(42-77)$ & $63(35-83)$ \\
Conventional treatment - mean age (range), years & 73 & $71(66-77)$ & $74(66-82)$ \\
TORS treatment - mean age (range), years & 42 & $42(42)$ & $55(35-83)$ \\
TORS TE + panscopy with biopsy, $n$ & 6 & 1 & 5 \\
Conventional TE + panscopy with biopsy, $n$ & 8 & 4 & 4 \\
\hline
\end{tabular}

TORS, transoral robotic surgery; TE, tonsillectomy.

used as primary treatment at high doses, followed by additional treatment such as radiotherapy [1].

Since 2009, Da Vinci's transoral robotic surgery (TORS) has been used in Europe, primarily for excision of smaller (T1-T2) oropharyngeal squamous cell carcinoma [2]. TORS gives a good 3D HD visualization of the operating field, enabling precise resection/biopsy of the suspected tumor area. The method has many advantages over conventional open surgery, where a so-called mandible split - dividing the lower jaw in order to reach tumor areas far back in the neck - is often performed. There is also an economic aspect, as the need for intensive care and hospitalization is considerably lower for patients treated with TORS than for those having had open surgery [2].

Studies have shown that TORS as monotherapy has the same - if not better - treatment results for T1-T2 cancer in the oropharynx compared to radiotherapy [3]. Also, in the event of a recurrence there is still the possibility of treating the patient with radiotherapy. Thus, there is lower treatment-related morbidity with TORS while one attains comparable oncological results to those of open surgery or chemotherapy [3].

In addition to improving survival, cancer physicians have increasingly focused on quality of life (QoL) issues, and there are several studies comparing different treatment modalities with respect to QoL [4]. The European Organization for Research and Treatment of Cancer (EORTC) was founded in 1962 as a nonprofit international organization, with the goal of developing and stimulating cancer research in Europe through major multidisciplinary prospective multicenter studies. In 1974, the EORTC's central data center was formed, which is now involved in all aspects of phase II and III clinical cancer trials. Six years later, in 1980, the EORTC QoL group was started, and in 1986, a project was initiated to develop a program for evaluating the QoL of patients participating in clinical cancer trials. This led to the development of the Quality of Life Questionnaire (QLQ)-C30 (online suppl. Table 1; for all online suppl. material, see www.karger.com/doi/10.1159/000509743), a cancer-specific questionnaire validated and used in several studies of head and neck cancer patients. The QLQ-C30 consists of a symptom scale, a functional scale, and a global health status/QoL scale. Its reliability and validity has been tested on, among others, 305 patients with lung cancer from 13 different countries [5].

The EORTC QLQ-H\&N35 [6] (online suppl. Table 2) was designed to be used in conjunction with the QLQ-C30, and it consists of 35 questions divided into 7 sections specific to patients with head and neck cancer. Its reliability and validity were tested by, among others, Bjordal and coworkers.

Postoperative pain can be assessed with a so-called visual analog scale (VAS) [7], where the patient marks her/his pain level between two endpoints. According to the US National Institutes of Health, this is the most reliable indicator of the incidence and intensity of pain [8]. The VAS has been shown to have excellent reliability and validity [9]. The Hospital Anxiety 
and Depression Scale (HADS) is another widely used self-assessment, yielding an estimate of the patient's anxiety and depression [10].

As the use of TORS is increasing in most ENT clinics, we wanted to look at the effects of this treatment from the patients' perspective and map advantages and disadvantages over conventional surgery. In this pilot study, the well-established and -validated questionnaires described above were used [11].

\section{Subjects and Methods}

A total of 14 patients with suspicion for oro- and hypopharyngeal squamous cell carcinomas $(\mathrm{T} 1, \mathrm{~T} 2)$ during the year 2018 were included in the study. The TORS procedure in this study consisted of tonsillectomy and panscopy with biopsy according to the standard protocol, compared to standard tonsillectomy and panscopy with biopsy. Exclusion criteria were mouth opening $<2.5 \mathrm{~cm}$; oro- and hypopharyngeal cancer $>\mathrm{T} 2$; previous treatment with head and neck surgery affecting swallowing, the voice or speech ability; previous radiation to the head and neck area; medical or neurological conditions affecting cognition, speech or swallowing; being under palliative treatment; and having other types of head and neck cancer. The patients were consecutively included, comprising 5 men (36\%) and 9 women (64\%). The total mean age was 64 years (35-83). For the gender and age distribution in the two groups, see Table 1.

All patients included in the study had been treated or were under treatment at the ENT Clinic at the University Hospital in Umeå, Sweden. Data were collected during regular examination and follow-up of the patients. This study was not randomized, due to its limited number of participants. A written information letter and the questionnaires were sent to the patients postoperatively, and validated questionnaires were used as a basis for monitoring QoL [11].

\section{Questionnaires}

The QLQ-C30 consists of a symptom scale (fatigue, nausea/vomiting, pain, shortness of breath, sleep problems, loss of appetite, constipation, diarrhea, and financial difficulties), a functional scale (physical, role, emotional, cognitive, and social), and a global health status/ QoL scale (patient's total health and QoL during the past week). The score obtained from each factor is translated into a scale of $0-100$, where a high score on the symptom scale indicates poorer health-related QoL (HRQoL), while high values on the global function scales indicate a better HRQoL $[12,13]$.

The EORTC QLQ-H\&N35 (online suppl. Table 2) is designed to be used in conjunction with the QLQ-C30, and it consists of 35 questions divided into 7 sections specific to patients with head and neck cancer, such as pain, swallowing, speech, mind, social eating, social contact and sexuality, and how patients experienced these sections/symptoms during the past week. Each section consists of single or multiple questions. High values on the symptom scale indicate lots of symptoms/problems.

Both the QLQ-C30 and the QLQ-H\&N35 contain questions to be answered on a 4-grade category scale. The answer options are $1=$ not at all, $2=$ little, $3=$ some, and $4=$ much. Assessment of global health status is performed via a 7-degree category scale, where $1=$ very poor and 7 = excellent. For the QLQ-H\&N35 there are also symptom questions answered with yes/no. When analyzing the questionnaire, all points on the numerical scale are converted to a 0-100 point scale according to the EORTC scoring manual. The questions in the sections concern different physical functions and different symptoms associated with head and neck cancer. For examples, see Table 2.

\section{Karger'}


Table 2. Function and symptoms (QLQ-C30 and QLQ-H\&N35)

\begin{tabular}{ll}
\hline Function and symptoms & Example \\
\hline Physical function & Carry heavy things, take walks \\
Role function & Limited ability to perform work or daily activities \\
Emotional ability & Anxiety or feeling depressed \\
Cognitive ability & Memory ability \\
Social function & Effect of the patient's physical or medical condition on family life \\
Fatigue & Needing rest, feeling tired and/or weak \\
Pain & Pain in the mouth, jaw, or throat \\
Shortness of breath & Being out of breath \\
Insomnia & Difficulty sleeping \\
Financial difficulties & Whether the patients' physical or medical condition has caused them \\
& financial difficulties \\
Speech difficulties & Being hoarse, having trouble talking \\
Social eating & Difficulty eating before the family or other people, enjoying the meals \\
Sexuality & Issues related to desire and sexual interest \\
Mouth opening & Having trouble opening the mouth \\
Global health & Experience/perception of overall health and quality of life in the past \\
Pain medication & week \\
\hline
\end{tabular}

The VAS is used for assessing postoperative pain using a straight line with endpoints that define the maximum limits as "no pain at all" and "pain as bad as it can be." The patient marks her/his pain level between the two endpoints. The HADS is a self-assessment of the patient's experienced level of anxiety and depression [10].

\section{Statistical Analysis}

Statistical analysis was performed using IBM SPSS statistics software, version 25.0. To analyze the TORS group and the conventional group, a Mann-Whitney U test was conducted. Probabilities of $<0.05$ were accepted as significant.

\section{Results}

\section{Questionnaire: Symptom Scales}

An evaluation of the symptom scales included in the questionnaires showed a higher pain score for patients treated with TORS compared to the conventional procedure. The difference was, however, not significant (Fig. 1). The median scores for the other factors were either fairly similar or showed only slight differences (Fig. 1).

\section{Questionnaire: Functional Scales}

In the QLQ-C30, high functional scale scores represent a high level of function. Cognitive, emotional, and physical functions had about the same spread in the TORS group and the conventional group. In role function, the TORS group showed a higher median level, although not significantly so, and the spread was bigger in the conventional group. In social function, the patients treated with TORS had a wider spread, albeit with an equal median in the two groups (Fig. 2). Looking at global health, the TORS group showed a higher median level, indicating better HRQoL, while the spread was bigger in the conventional group (Fig. 3). Regarding the proportion of complications 30 days postoperatively, the TORS group showed a higher 


\section{Case Reports in Oncology}

Fig. 1. Observations for symptoms (more than one item). No factor was significantly different between the two groups (either treated with TORS or the conventional procedure). FA, fatigue; NV, nausea and vomiting; $\mathrm{PA}$, pain; $\mathrm{SCH}$, social contact; $\mathrm{SEH}$, senses; $\mathrm{SOH}$, social eating; SPH, speech; SWH, swallowing; SXH, sexuality; TORS, transoral robotic surgery.

Fig. 2. Boxplot for the functional scale. Score points in each group and category. None of the functions showed any significant difference between patients treated with TORS and those treated with the conventional procedure. $\mathrm{CF}$, cognitive function; EF, emotional function; PF2, physical function; RF2, role function; SF, social function; TORS, transoral robotic surgery.

Fig. 3. Boxplot for general health condition, showing a higher median value, indicative of better health-related quality of life, for the TORS group, whereas a bigger spread was seen in the group having undergone the conventional procedure. TORS, transoral robotic surgery.

\begin{tabular}{l|l}
\hline Case Rep Oncol 2020;13:1295-1303 \\
\hline DOI: 10.1159/000509743 & $\begin{array}{l}\text { @ 2020 The Author(s). Published by S. Karger AG, Basel } \\
\text { www.karger.com/cro }\end{array}$ \\
\hline
\end{tabular}
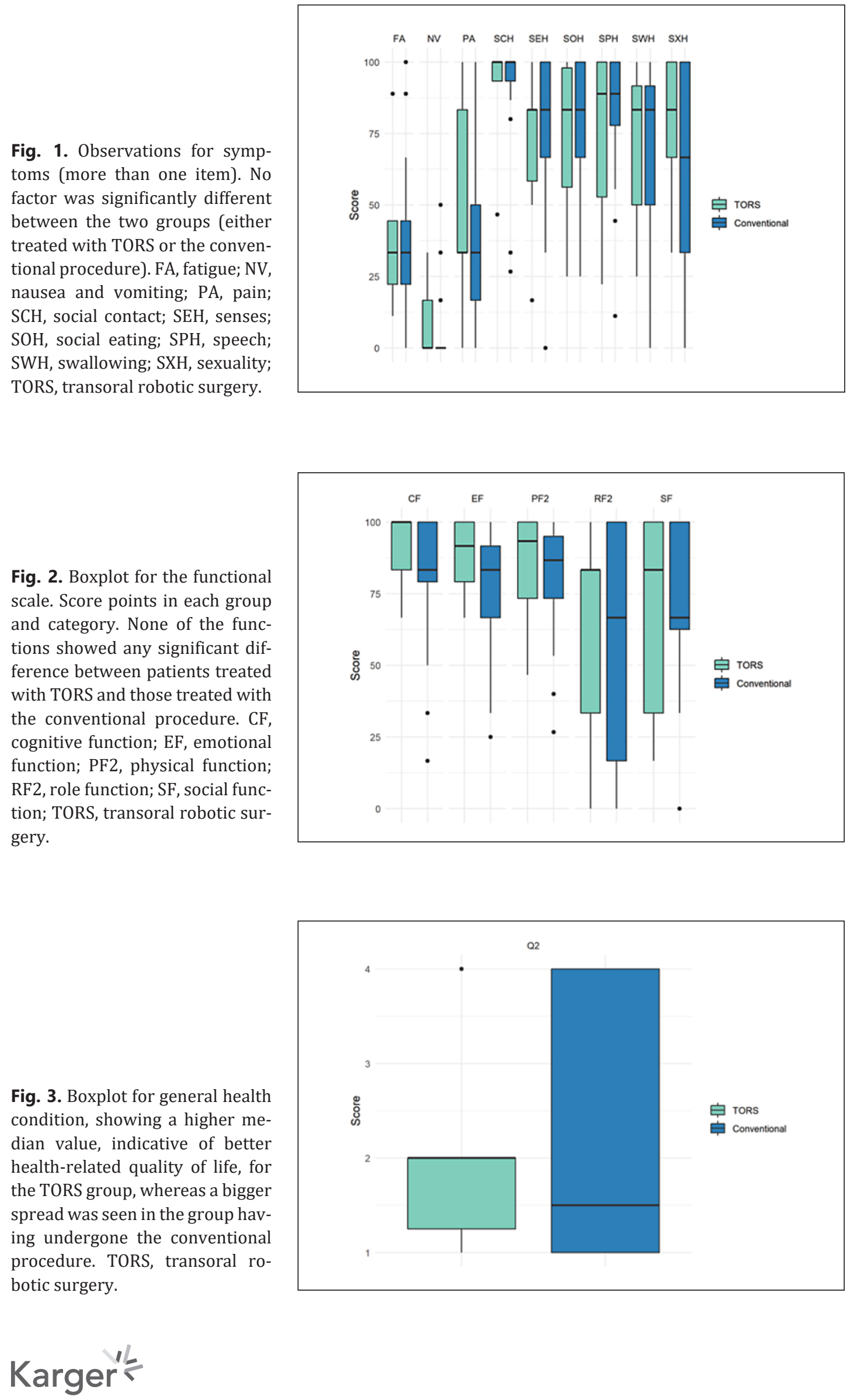


\section{Case Reports in Oncology}

Fig. 4. Complications 30 days after surgery. Bars represent the proportion of patients with complications in the following areas: TE2 = contact with the healthcare system because of bleeding in the throat; TE3 = admitted to hospital due to bleeding from the throat; TE4 = additional operations performed due to bleeding; TE5 = infection occurred during the care period or within 30 days postoperatively; TE6 = contact with the healthcare system because of infection; TE7 = received antibiotic treatment because of infection; TE8 = contact with the healthcare system because of pain after surgery; TE9 = how many days after surgery did you take painkillers; TE12 = information received regarding the operation and experience after - did it meet your expectations; TORS, transoral robotic surgery.

Fig. 5. Boxplot for the VAS score in each group and category. VAS1 = pain score on postoperative day 1; VAS3 = pain score on postoperative day 3; VAS5 = pain score on postoperative day 5 ; VAS7 = pain score on postoperative day 7. VAS, visual analog scale.

\begin{tabular}{l|l}
\hline Case Rep Oncol 2020;13:1295-1303 \\
\hline DOI: 10.1159/000509743 & $\begin{array}{l}\text { @ 2020 The Author(s). Published by S. Karger AG, Basel } \\
\text { www.karger.com/cro }\end{array}$ \\
\hline
\end{tabular}
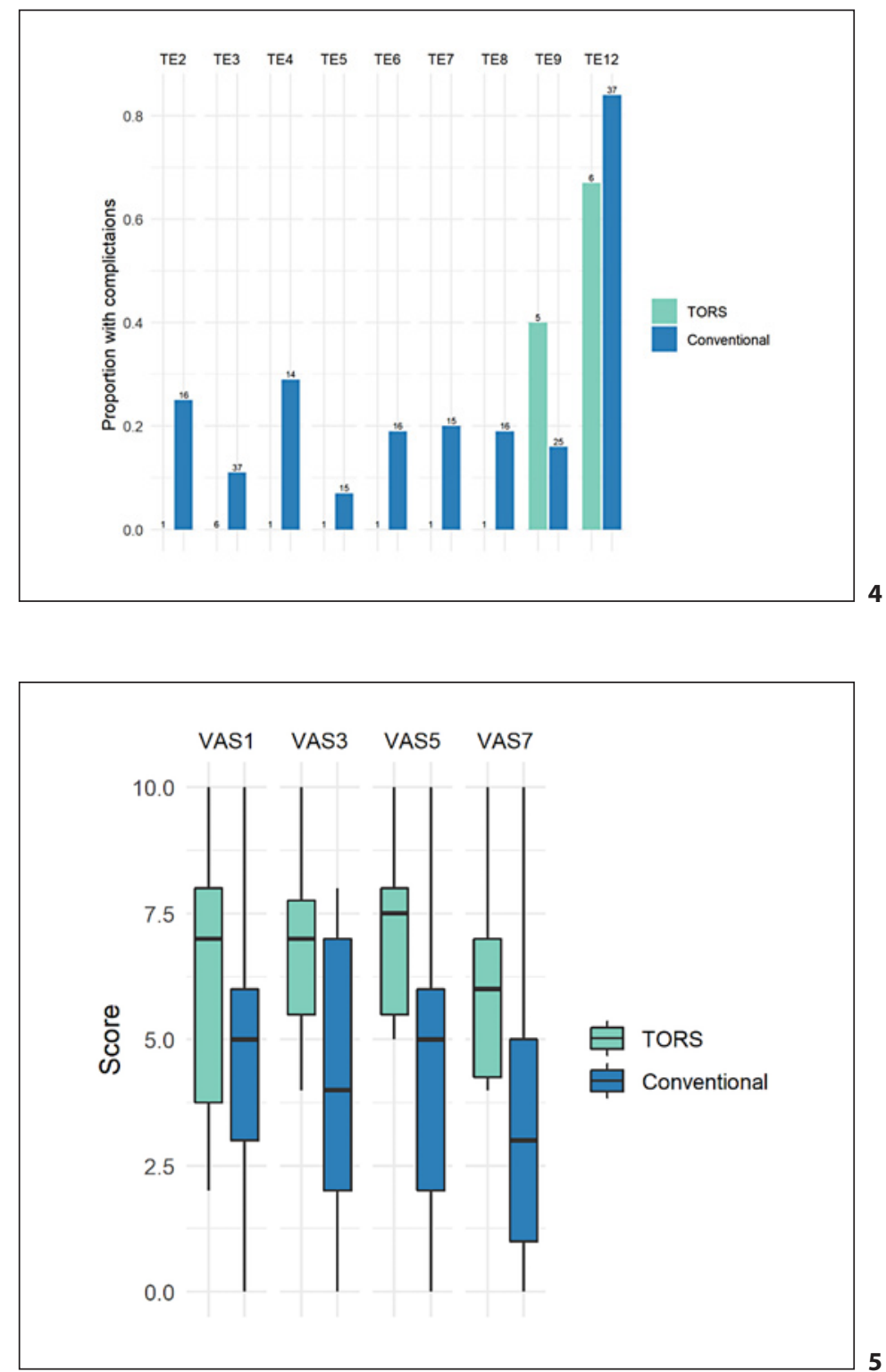

need for painkillers, whereas the pre- and postoperative information given did not meet the expectations of the patients in the conventional group (Fig. 4).

Visual Analog Scale

The scores for postoperative pain during the first week showed slightly higher pain among patients treated with TORS (Fig. 5).

\section{Discussion}

The use of TORS has many advantages over conventional surgery, including the absence of external incisions, maintenance of normal surrounding structures, a shorter operating time, and a lower number of hospital days [14], resulting in better function than with the conventional procedure. 
In recent years, the focus has not only been on increased survival but also on patient QoL, and our study is the first to describe outcomes of treatment with TORS compared to conventional surgery. The patients in the TORS group reported higher levels of pain and also used painkillers for a longer period than those having had conventional treatment. Previous studies have shown sex-based differences in the perception, management and reporting of pain, with women reporting higher levels of pain, increased pain sensitivity, and higher functional effects [15]. The fact that the majority of the patients in our TORS group were women (5 out of 6 by random allocation) could thus help explain the differences in postoperative pain that we observed between the two groups. The mean age in the group receiving conventional treatment was higher (73 years) than in the TORS group (42 years). As there is evidence of episodic memory decline with age [16], this could explain why the pre- and postoperative information given did not meet the expectations of the patients in this group.

Human papilloma virus status is routinely used in TNM staging of oro-hypopharyngeal tumors. However, as QoL-derived survival predictions do not depend on a high human papilloma virus risk, this factor was not taken into account here [17].

Combination therapy is an effective way of treating cancer disease and reducing local disease, but it also increases the risk of complications, reduces function, and affects QoL. In their study, Leonhardt et al. [18] concluded that TORS and adjuvant therapy caused a temporary decrease in scores on several scales at 6 months, returning to baseline at 12 months with all patients, largely accreditable to adjuvant chemotherapy. The acute toxicity of chemoradiation is to blame for loss of function and QoL, whereas treatment with TORS alone does not considerably affect swallowing function.

In the present study, 14 patients were included, with a mean age of 64 years, a fact that, however, did not influence the results of the questionnaires. Both the QLQ-C30 and the QLQ-H\&N35 not only are valid and reliable but also deal with general health and specific head and neck-related questions, a combination providing a comprehensive assessment. The HADS included in this study is a self-assessment scale measuring anxiety/depression intended to be used for screening purposes. Even if the scores did not prove high enough to be classified as depression or anxiety, patients treated with the conventional procedure experienced more anxiety and signs of depression. This is a fact to be taken into consideration, as it has been shown that psychiatric distress persists long after treatment [19].

Together with previously published data on the clinical advantages of using TORS for excision of T1-T2 oropharyngeal squamous cell carcinomas - such as good visualization of the operating field, lower treatment-related morbidity, etc. - our present data also show advantages of the TORS technique from the patients' perspective. Even if patients treated with TORS are in need of more painkilling treatment, they cope better with the long-term effects of treatment, as judged by self-assessment of anxiety and depression, which could be due, for example, to the lack of effects caused by open surgery.

To our knowledge, this is the first study comparing patients' experiences of treatment with TORS and the conventional procedure. Even if a limited group of patients were included in this pilot study, our data highlight the advantages of using TORS.

\section{Statement of Ethics}

This study was approved by the local ethics committee (Regionala Etikprövningsnämnden, Umeå; Dnr 2018-105-31M). The patients consented to this study in writing and orally prior to the operation.

\section{Karger'}




\section{Conflict of Interest Statement}

There are no conflicts of interest reported by any of the authors.

\section{Funding Sources}

The Swedish Cancer Society funded this study (contract No. 1805 42).

\section{Author Contributions}

A.M. Salehi planned the study, analyzed the data, and wrote the manuscript; L. NorbergSpaak planned the study and wrote the manuscript; T. Wilms wrote the manuscript; S. Vallin analyzed the data; L. Boldrup wrote the manuscript; N. Sgaramella analyzed the data and wrote the manuscript; M. Majlesi analyzed the data; S. Nezafat analyzed the data; K. Nylander wrote the manuscript. All authors read and approved the submitted version of the manuscript.

\section{References}

1 Lörincz BB, Knecht R. TORS for head and neck squamous cell carcinoma (HNSCC): the Hamburg experience. B-ENT. 2015;Suppl 24:33-6.

2 Lörincz BB, Möckelmann N, Busch CJ, Knecht R. Functional outcomes, feasibility, and safety of resection of transoral robotic surgery: single-institution series of 35 consecutive cases of transoral robotic surgery for oropharyngeal squamous cell carcinoma. Head Neck. 2015;37(11):1618-24.

3 Cosmidis A, Rame JP, Dassonville 0, Temam S, Massip F, Poissonnet G, et al. T1-T2 N0 oropharyngeal cancers treated with surgery alone. A GETTEC study. Eur Arch Otorhinolaryngol. 2003;261(5):276-81.

4 Achim V, Bolognone RK, Palmer AD, Graville DJ, Light TJ, Li R, et al. Long-term functional and quality-of-life outcomes after transoral robotic surgery in patients with oropharyngeal cancer. JAMA Otolaryngol Head Neck Surg. 2018;144(1):18-27.

5 Aronson NK, Ahmedzai S, Bergman B, Bullinger M, Cull A, Duez NJ, et al. The European Organization for Research and Treatment of Cancer QLQ-C30: a quality-of-life instrument for use in international clinical trials in oncology. J Natl Cancer Inst. 1993;85(5):365-76.

6 Bjordal K, Ahlner-Elmqvist M, Tollesson E, Jensen AB, Razavi D, Maher EJ, et al. Development of a European Organization for Research and Treatment of Cancer (EORTC) questionnaire module to be used in quality of life assessments in head and neck cancer patients. EORTC Quality of Life Study Group. Acta Oncol. 1994;33(8): 879-85.

7 Haefeli M, Elfering A. Pain assessment. Eur Spine J. 2006;15 (Suppl 1):S17-24.

8 Ferraz MB, Quaresma MR, Aquino LR, Atra E, Tugwell P, Goldsmith CH. Reliability of pain scales in the assessment of literate and illiterate patients with rheumatoid arthritis. J Rheumatol. 1990;17(8):1022-4.

9 O'Rourke D. The measurement of pain in infants, children, and adolescents: from policy to practice. Phys Ther. 2004;84(6):560-70.

10 Zigmond AS, Snaith RP. The Hospital Anxiety and Depression Scale. Acta Psychiatr Scand. 1983;67(6):361-70.

11 Bjordal K, Kaasa S, Mastekaasa A. Quality of life in patients treated for head and neck cancer: a follow-up study 7 to 11 years after radiotherapy. Int J Radiat Oncol Biol Phys. 1994;28(4):847-56.

12 Fayers PM, Aaronson NK, Bjordal K, Sullivan M. EORTC QLQ-C30 scoring manual. Brussels: European Organization for Research and Treatment of Cancer Study Group on Quality of Life; 1995.

13 Fayers PM, Aaronson NK, Bjordal K, Sullivan M. EORTC QLQ-C30 scoring manual. 3rd ed. Brussels: European Organisation for Research and Treatment of Cancer Care; 2001.

14 O'Malley BW Jr, Weinstein GS, Snyder W, Hockstein NG. Transoral robotic surgery (TORS) for base of tongue neoplasms. Laryngoscope. 2006;116(8):1465-72.

15 Bartley EJ, Fillingim RB. Sex differences in pain: a brief review of clinical and experimental findings. Br J Anaesth. 2013;111(1):52-8.

16 Sommer VR, Fandakova Y, Grandy TH, Shing YL, Werkle-Bergner M, Sander MC. Neural pattern similarity differentially relates to memory performance in younger and older adults. J Neurosci. 2019;39(41):80898099. 
17 Aarstad HJ, Østhus AA, Aarstad HH, Lybak S, Aarstad AKH. General health-related quality of life scores from head and neck squamous cell carcinoma patients obtained throughout the first year following diagnosis predicted up to 10-year overall survival. Eur Arch Otorhinolaryngol. 2018;275(1):207-17.

18 Leonhardt FD, Quon H, Abrahão M, O’Malley BWJr, Weinstein GS. Transoral robotic surgery for oropharyngeal carcinoma and its impact on patient-reported quality of life and function. Head Neck. 2012;34(2):146-54.

19 Bjordal K, Kaasa S. Psychological distress in head and neck cancer patients 7-11 years after curative treatment. Br J Cancer. 1995;71(3):592-7. 